\title{
DEMOGRAFÍA DE PALAEMONETES ARGENTINUS NOBILI (DECAPODA NATANTIA) EN LA LAGUNA CHASCOMUS, BUENOS AIRES, ARGENTINA. SUPERVIVENCIA, MIGRACIONES, BIOMASA Y FECUNDIDAD .*
}

\author{
A. Rodrigues Capítulo y L. R. Freyre \\ Instituto de Limnología "Dr. Raúl A. Ringuelet", CC 712, CP 1900-La Plata, Buenos Aires, Argentina
}

Palabras clave: Palaemonetes argentinus, demografía, lagunas pampásicas, Argentina.

Keywords: Palaeomonetes argentinus, demography, pampasic lakes, Argentina.

\begin{abstract}
DEMOGRAPHY OF PALAEMONETES ARGENTINUS NOBILI (DECAPODA NATANTIA). SURVIVAL, MIGRATIONS, BIOMASS AND FECUNDITY.

Numerical changes of a P. argentinus population in Chascomús Pond (Buenos Aires province, Argentina) are analyzed here based on the application of exponential survival model. Observed variations in density and biomass during the year, were attributed to internal migrations within the pond. The description of numerical variations including mortality and local migrations was explained by the following equation: $N(t)_{i j}=10^{z} N_{o} i j e^{-z j\left(t-t_{n i}\right)}$, where $i=$ cohort, $j=$ sex, $t=$ time, $N_{o}=$ initial number, $z=$ instantaneous rate of mortality, and $\mathrm{E}=$ a Fourier polynomial of one order. The biomass to which each sex and cohort contributed was also discriminated. The percentage of embrionated females during annual cycle and spawning season are recorded. The first mean size was estimated at approximately $10,9 \mathrm{~mm}$ of the caparison-rostrum corresponding to one year old individuals. In order to express fecundity (F) in relation with the length of caparison-rostrum (CR) the following equation was used: $\mathrm{F}=0.074 \mathrm{CR}^{2.014}$. The fecundity of each one of the three cohorts was analyzed during the three spawning season.
\end{abstract}

\section{INTRODUCCIÓN}

El camarón Palaemonetes argentinus (Caridea, Palaemonidae) es una especie sudamericana que vive exclusivamente en agua dulce. Se encuentra desde el sur de Brasil (Río Grande do Sul) hasta la región centrolitoral de Argentina (Córdoba, Mendoza, Delta del Paraná, Río de la Plata (RINGUELET, 1949, BOSCHI, 1981, RODRIGUES CAPÍTULO \& FREYRE, 1989, SCHULDT \& RODRIGUES CAPÍTULO, 1985) y tiene su límite de distribución sur en la Provincia de Buenos Aires, poblando charcas artificiales, arroyos y lagunas pampásicas.

Por su abundancia numérica y protagonismo en las cadenas tróficas de ambientes pampásicos varios autores han dedicado estudios tendientes al conocimiento de su biología y desarrollo larval (BOSCHI, 1961, MENU-MARQUE, 1973, GOLDSTEIN \& LAURTA DE CIDRE, 1974), metabolismo energético (RODRIGUES CAPÍTULO \& FREYRE, 1979), o sus relaciones tróficas (DESTEFANIS \& FREYRE, 1972, RINGUELET et al., 1980, SCHULDT(1980 a, b, 1981) complementóinformación sobre el desarrollo gonadal de las hembras de esta especie. En relación con su dinámica poblacional RODRIGUES CAPÍTULO \& FREYRE (1989) dieron a conocer los primeros resultados demográficos de $P$. argentinus en la Laguna Chascomús basados en muestreos quincenales. Se describieron en esas contribuciones la presencia de tres cohortes anuales, correspondientes a los periodos reproductivos de octubre, diciembre y enero, donde se analizó el crecimiento en longitud y peso de las mismas. Posteriormente RODRIGUES CAPÍTULO (1989) sumarizó, en tablas de vida ecológicas, algunos datos de supervivencia, expectativa media de vida y reproducción de esta especiecon el material provenientede los mismos muestreos.

En el presente trabajo se describe la supervivencia de tres cohortes de cada sexo, su biomasa y la fecundidad así como también las migraciones poblacionales locales que ocurren en la laguna a lo largo del ciclo anual. 


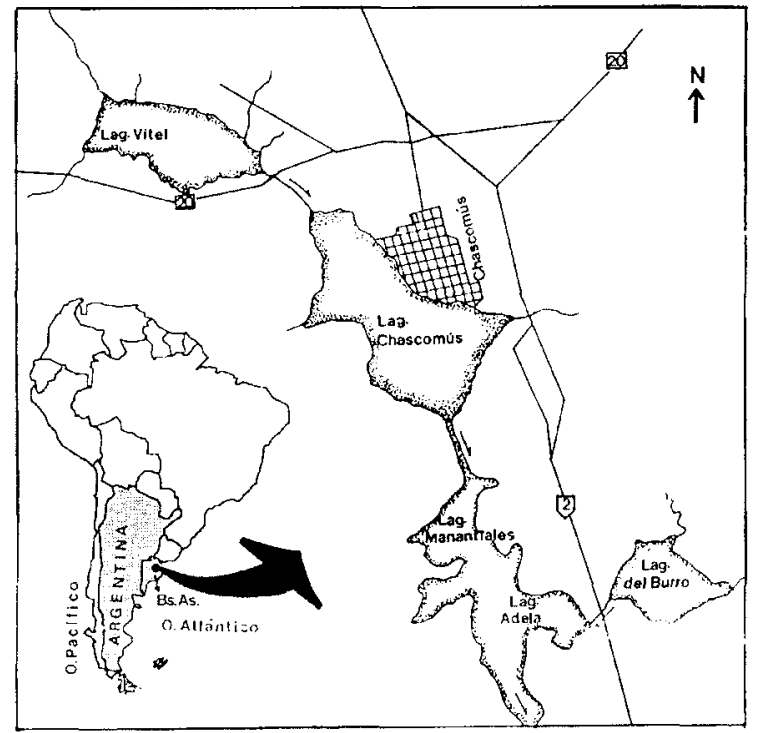

FIGURA 1. Localización de la Laguna Cascomún en la Provincia de Buenos Aires, Argentina

FIGURE I Location of the Chascomús Pond in the Buenos Aires Province, Argentine

\section{MATERIAL Y MÉTODOS}

Área de estudio (Fig. 1).

La laguna Chascomús está ubicada a $58^{\circ} 00^{\prime}$ W y $35^{\circ} 35^{\prime} \mathrm{S}$, en la Provincia de Buenos Aires, Argentina. Tiene una superficie total de $3012.9 \mathrm{Ha}$; longitud máxima de $9570 \mathrm{~m}$ con rumbo NW-SE; ancho máximo $6260 \mathrm{~m}$ y ancho medio de 3148 $\mathrm{m}$; perímetro o longitud de línea de costa $28120 \mathrm{~m}$; desarrollo dc linea de costa: 1.44; volúmen total $47015300 \mathrm{~m}^{3}$; profundidad máxima: $1.90 \mathrm{~m}$; profundidad media: $1.53 \mathrm{~m}$; superficie cubierta por juncales (Schoenocleprus californicus): 393 Ha (DANGAWS, 1976). Forma parte del sistema de lagunas encadenadas originadas, según RINGUELET (1962) a partir dc un curso fluvial preexistente, o bien según otros autores a erosión eólica hasta el nivel freático y luego unidas en períodos relativamente recientes (fines del Platense fluvial) por captura de las aguas estancadas por acción del Río Salado. Presenta barrancas en casi toda la línea de costa. Tiene como cursos afluentes los arroyos Vitel, Valdés y San Felipe y como efluente principal el arroyo Girado. El fondo está constituido por sedimentos Iimoarenosos unimodales en la zona costera y de sedimentos limosos polimodales en la zona profunda. En varios sectores existen afloramientos de tosca. La temperatua media es de $14^{\circ} \mathrm{C}$ con una media mensual mínima de $5^{\circ} \mathrm{C}$ en julio y media inensual máxima de $18.8^{\circ} \mathrm{C}$ en enero.

El sector muestreado correspondió a la zona Oeste de la laguna en el lugar conocido como Monte Brown, con fondo de toscas y libre de macrófitas. Este área se encuentra cubierta la mayor parte del año por algas filamentosas (Clorofitas).

Muestreos. Se realizaron un total de 33 muestreos quincenales en la Laguna Chascomús en el período 1974-1976. Estos se practicaron con una red de arrastre de $2 \mathrm{~mm}$ de abertura de malla (SENDRA \& FREYRE, 1978, RODRIGUES CAPÍTULO \& FREYRE, 1989) lanzada gradualmente desde una embarcación ubicada a 30 metros de la orilla y recogida por dos personas en forma abierta y vertical desde la costa, (FREYRE, 1975). El área de muestreo barrida fue dc $420 \mathrm{~m}^{2}$. Cada uno de estos lances fué considerado como una unidad de esfuerzo. La metodología general para el presente estudio se basó en los criterios de RICKER (1968. 1973) y GULLAND (1971).

Supervivencia. A partir de los datos de abundancia obtenidos de la descomposición poliinodal para machos y hembras (RODRIGUES CAPITULO \& FREYRE, 1989) se intentó la aplicación del modelo exponencial de supervivencia: $\mathrm{Nt}=\mathrm{N}_{\mathrm{O}} \mathrm{e}^{-\mathrm{Z}\left(\mathrm{t}-\mathrm{t}_{\mathrm{n}}\right)}, \ldots \ldots \ldots \ldots \ldots \ldots \ldots \ldots \ldots \ldots \ldots \ldots \ldots \ldots \ldots \ldots$

donde $\mathrm{N}_{\mathrm{o}}$ : número inicial, z: tasa instantánea de mortalidad, $t_{n}$ : tiempo de nacimiento. Para el ajuste del modelo a los datos se utilizó la expresión logarítmica:

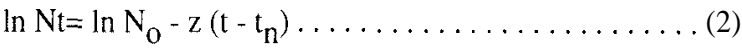

Para cada una de las cohortes se calculó la siguiente expresión:

$\ln \mathrm{Nt}-\ln \mathrm{NG}=-\mathrm{z}\left(\mathrm{t}-\mathrm{t}_{\mathrm{n}}+\operatorname{In} \mathrm{N}_{\mathrm{o}} \ldots \ldots \ldots \ldots \ldots\right.$ (3)

donde ln NG: es el logaritmo ncperiano de la inedia geométrica de los $\mathrm{N}$ observados. Para la suma de las tres cohortes se estimaron los valores de $\ln \mathrm{N}_{\mathrm{o}}$ y z como constantes de ajuste. Por último se estimó para cada cohorte la siguiente ecuación aplicando proporcionalidades:

$\ln \mathrm{N}_{\mathrm{oi}}=\ln \mathrm{N}_{\mathrm{o}}+\ln \mathrm{NG}_{\mathrm{i}}$

Se obtuvieron de esta manera las tasas de mortalidad para cada sexo y los respectivos valores de $\mathrm{N}_{\mathrm{O}}$ para los machos y hembras de cada cohorte.

Migraciones locales. Los ajustes del modelo de supervivencia antes comentado (1) muestran que una parte importante de la varianza quedaba sin explicar. Para la explicación de estas discrepancias se siguió la hipótesis planteada por SENDRA \& FREYRE (1981) que atribuyen los cambios de densidad en poblaciones de peces dc la laguna Chascomús a migraciones internas o locales. De esta manera se obtuvo el índice:

$$
\mathrm{K}=\sum_{1} \mathrm{NO}_{\mathrm{i}}(\mathrm{t}) / \sum_{\mathrm{i}} \mathrm{N}_{\mathrm{i}}(\mathrm{t})
$$


donde $\mathrm{NO}_{\mathrm{i}}(\mathrm{t})$ es el número de individuos observados de la cohorte $i$ de la fecha $t$, y $N_{\mathrm{i}}(\mathrm{t})$ el número estimado para la misma cohorte y fccha. Este índice $\mathrm{K}$ es una medida del grado de concentración o dilución de los individuos en la estación muestreada para la fecha $\mathrm{t}$ y tendrá un comportamiento periódico anual. Se efectuó el análisis armónico del Ig K, cantidad que en adelante se denoininó epsilón (E).

Resulta entonces de lo hasta aquí analizado que una buena descripción de la densidad en el área de muestreo que incluyera las variaciones por mortalidad y migraciones estaría dada por:

$$
\begin{aligned}
& \mathrm{N}(\mathrm{t})\left\{\mathrm{j}=10^{\varepsilon} \text { Noij } \mathrm{e}^{-\mathrm{zj}\left(\mathrm{t}-\mathrm{t}_{\mathrm{m}}\right)}, \ldots \ldots \ldots \ldots \ldots \ldots \ldots \ldots \ldots \ldots \ldots\right. \\
& \text { donde } \mathrm{i}=\text { cohorte, } \mathrm{j}=\text { sexo, y êpsilon (E) está dado por: } \\
& \varepsilon=\mathrm{A}_{\mathrm{O}}+\mathrm{A}_{1} \cos (360 \mathrm{t})+\mathrm{Bi} \operatorname{scn}(360 \mathrm{t}) \ldots \ldots \ldots \ldots
\end{aligned}
$$

Biomasa. Con el propósito de cstiinar a lo largo del año la biomasa (BM) de la población de P. argentinus referida a la unidad de esfuerzo considerada, en la estación tosca, se calculó el peso húmedo de los camarones de acuerdo a la ecuación:

$W=c L^{n}$ descripta en RODRIGUES CAPÍ TULO \& FREYRE (1989) donde W es el peso húmedo, L es la longitud del caparazón hasta la punta anterior del rostro y $n$ es la constante de proporcionalidad. El peso húmedo fué multiplicado por el número de individuos presentes en la estación tosca de la edad correspondiente $\left(\mathrm{K} \mathrm{N}_{\mathrm{t}}\right)$ para todas las cohortes de cada sexo. Se sumaron posteriormente los pesos parciales de los diferentes conjuntos (cohortes) en cada intervalo de tiempo ( $t=0,1$ años) y luego se procedió a sumar las biomasas que se presentan simultáneamente en un sólo ciclo anual ( $\mathrm{t}=0,05$ años). Se puso de manifiesto de esta manera el aporte por sexo de las cohortes de primavera, verano medio y verano avanzado en todo el año. Finalmente se halló la biomasa total de machos y hembras .

Fecundidad. Para la estimación del tamaño medio de primera maduración de las hembras se evaluó, a partir de la estructura de la población, el porcentaje de hembras ovígeras a lo largo del año elaborándose un histograma con dichos datos. Dado que podían observarse 3 valores máximos, éstos se descompusieron como suma de normales utilizando un método semicomputarizado según FREYRE (1981). Se obtuvieron así 3 curvas normales con sus correspondientes medias (fccha modal de cada desove), $s$ (la dispersión temporal del desove) y modal de cada desove), s (la dispersión temporal del desove) y $\mathrm{r}$ (coeficiente de correlación de la recta probabilística ajustada).

La fecundidad de los camarones se ha evaluado a partir del número de huevos por hembra ovígera en los momentos de desove. Sc examinaron con este propósito 83 hembras ovígeras correspondiendo 40 al primer desove, 18 al segundo y 25 al tercero. Se analizó posteriormente el número de huevos en función de la talla de las hembras en intervalos de $1 \mathrm{~mm}$ de la longitud del caparazón + rostro.

El cálculo de la fecundidad (F) se estimó según la expresión:

$\mathrm{F}=\mathrm{a} \mathrm{L}^{\mathrm{b}}$

donde a y b son constantes de proporcionalidad y L es la longitud del caparazón + el rostro.

Con el propósito de analizar la participación que tiene cada una de las cohortes en la fecundidad de la población de P. argentinus en la laguna Chascomús se observó la estructura de edades de las hembras en los períodos de puesta. Se correlacionó luego el tamaño de éstas con la cantidad de huevos sostenidos entre los pleópodos de acuerdo a la expresión (8) y se halló en base al gráfico de tamaño medio de primera maduración, llevado a escala probabilísitica normal, el porcentaje de participación en función de las tallas. Se estimó posteriormente el porcentaje de hembras ovígeras en las épocas reproductivas. El producto del número de hembras supervivientes en la época del desove (ecuación 6) por las dos proporciones anteriores, multiplicados por la Fecundidad de acuerdo a la ecuación (8) para cada clase de tamaño, permitió estimar el aporte total de cada cohorte en cada uno de los desoves y obtener, con la integración del conjunto, el número de embriones producidos para las próximas generaciones.

Se calculó además el peso formólico y volúmen medios de los huevos de P. argerztirzus para lo cual se pesaron 97 huevos y se midieron los ejes mayor y menor de 10 huevos estimándose su volúmen según la ecuación del elipsoide prolato.

$$
V=(\pi / 6) a b^{2}
$$

donde $a$ y b son respectivamente los ejes mayor y inenor del elipsoide.

\section{RESULTADOS Y DISCUSIÓN}

Supervivencia. De la aplicación de la expresión de supervivencia (1) se obtuvieron las siguientes ecuaciones para las diferentes cohortes de cada sexo: 
Hembras:

$$
\begin{aligned}
& N_{t 1}=96.02 e^{-2.204(t-0.817)} \\
& N_{t 2}=207.29 e^{-2.204(t-0.953)} \\
& N_{t 3}=68.60 e^{-2.204(t-1.078)}
\end{aligned}
$$

Con un coeficiente de correlación (r) para la forma logarítmica de la suma de cohortes $=-0,485$.

Machos:

$$
\begin{aligned}
& \mathrm{N}_{\mathrm{t} 1}=92.38 \mathrm{e}^{-1.566(\mathrm{t}-0.817)} \\
& \mathrm{N}_{\mathrm{t} 2}=81.54 \mathrm{e}^{-1.566(\mathrm{t}-0.953)} \\
& \mathrm{N}_{\mathrm{t} 3}=23.25 \mathrm{e}^{-1.566(\mathrm{t}-1.078)}
\end{aligned}
$$

Con un coeficiente de correlación ( $\mathrm{r}$ ) para la forma logarítmica de la suma de cohortes $=-0,284$. Los bajos valores del coeficiente de correlación fueron seguidamente explicados bajo la hipótesis de migraciones locales.

Si tenemos en cuenta las observaciones de supervivencia realizadas por MALLO \& BOSCHI (1982) para Peisos petrunkevitchi, una especie de camarón marino en las costas bonaerenses de Argentina, su longevidad (13-15 meses) es aproximadamente similar a la hallada para P. argentinus, pues si bien se estimó una mayor expectativa de vida para nuestra especie dulceacuícola, sólo unos pocos individuos llegan a sobrepasar estas edades.

Migraciones locales. Parte de la dispersión de los datos fue interpretada y explicada como concentraciones o diluciones en el área de estudio debidas posiblemente a migraciones o desplazamientos de los camarones hacia otras comunidades de la laguna en épocas definidas del año. Estas observaciones también fueron afirmadas por MALLO \& BOSCHI (1982) para Peisos petrunkevitchi. La observación de los valores que adopta el índice $\mathrm{K}$ mostró en líneas generales una dilución en los meses invernales y una concentración de la población en la época estival, destacándose una manifiesta coincidencia entre machos y hembras.

Se representó la media mensual de E y luego se ajustó a dichos datos un polinomio trigonométrico de Fourier (fig. 2, tabla 1) Se aceptó para E el ajuste de orden uno, aunque quizás otros armónicos, como por ejemplo el de orden cuatro, expliquen variaciones reales que deberían confirmarse con muestreos de mayor extensión en el tiempo. Un buen estimador de E resultó:

$$
E=0.224+0.486 \cos (360 t)+0.152 \operatorname{sen}(360 t)
$$

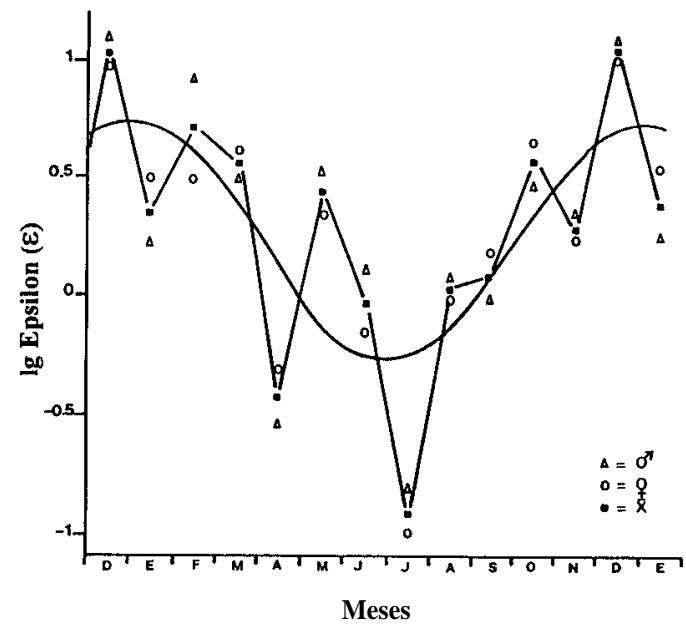

FIGURA 2. Valores épsilon ( $\varepsilon=1 \mathrm{~g} 10 \mathrm{k}$ ) para machos, hembras y promedios durante el año. La sinusoide (línea continua) corresponde a la aplicación a $\varepsilon$ del modelo de Founer.

FIGURE 2. Value of epsilon ( $\varepsilon=1 \mathrm{~g} 10 \mathrm{k}$ ) for male, and averages, during the year. The sinusoid (continued line) corresponds to the application to $E$ of the model of Fourier.

TABLA I. Constantes para diferentes órdenes de polinomio de Fourier y su porcentaje de variación explicada.

TABLE I. Constants for different orders of the Fourier polynomial, and percent of explained variation.

\begin{tabular}{cccc|}
\hline $\begin{array}{l}N^{0} \text { de orden } \\
\text { de } \varepsilon\end{array}$ & $A$ & $B$ & $\begin{array}{c}\% \text { de } \\
\text { variación } \\
\text { Explicada }\end{array}$ \\
\hline 0 & 0.2243 & & \\
1 & 0.4856 & 0.1523 & 46.64 \\
2 & 0.00195 & -0.0934 & 1.58 \\
3 & -0.0976 & 0.1606 & 6.36 \\
4 & 0.1946 & -0.2618 & 19.16 \\
5 & 0.1557 & 0.2414 & 14.85 \\
6 & 11.1844 & $1.7560 \mathrm{E}^{-12}$ & 12.25 \\
\hline
\end{tabular}

que explica un $47 \%$ de las desviaciones numéricas aún restantes. De esta manera se procedió a obtener $\operatorname{los} \mathrm{N}_{\mathrm{t}}$ estimados incluyendo e de acuerdo a la ecuación (7), para las tres cohortes de cada sexo. En la figura 3 se dispusieron los valores de $\mathrm{N}_{\mathrm{t}}$ para machos y hembras respectivamente y las correspondientes tallas (I-II-III), estimados según la ecuación (3) y constantes (8) de RODRIGUES CAPITULO \& FREYRE (1989).

Biomasa. Las estimaciones de la biomasa parcial en el área de estudio (banda costera con fondo de tosca) obtenida a partir del producto del peso medio individual, multiplicado por " $\mathrm{KN}$ "para cada cohorte, se representaron en la figura 4. La biomasa por sexo en un ciclo anual y la suma de ambas se representaron 

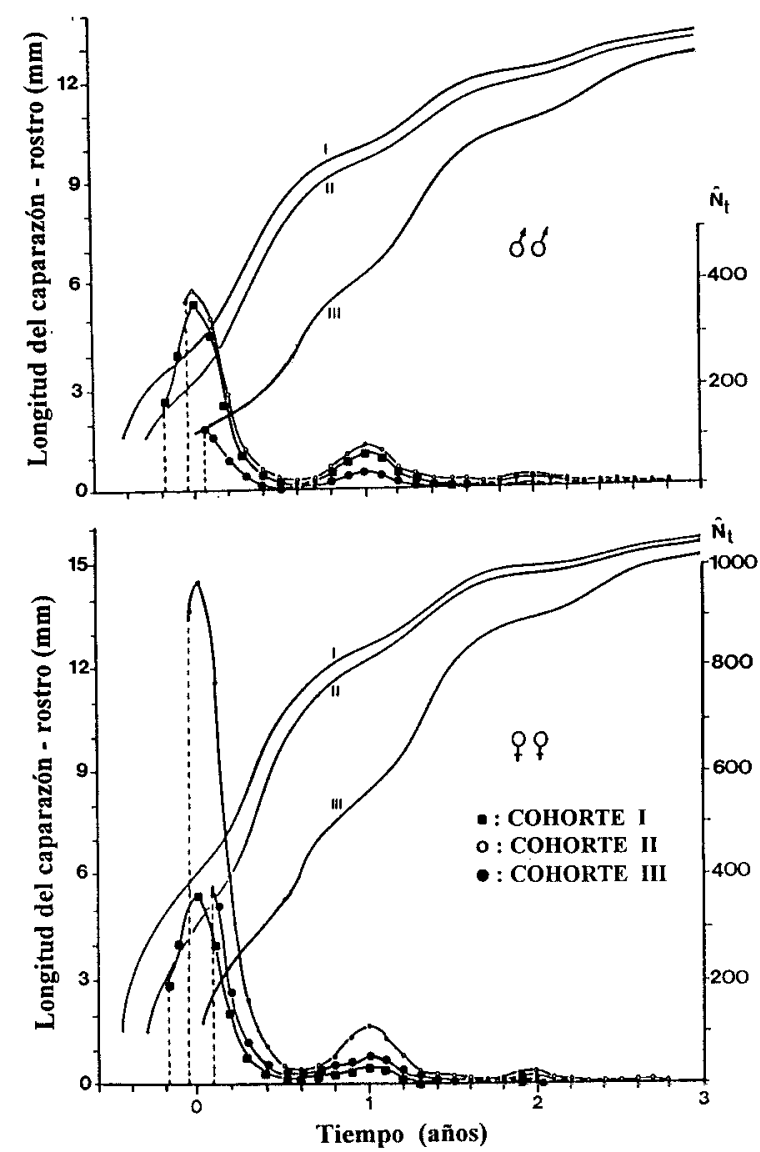

FIGURA 3. Supervivencia de $P$. argentinus (cohortes I, II y III) para machos y hembras respectivamente (escala derecha) desde el inicio hasta la dilución total de las cohortes. Curvas I, II y III: Tallas del CR (escala izquierda) en función del tiempo, según las modificaciones de RODRIGUES CAPÍTULO \& FREYRE, (1989) al modelo de Bertalanffy.

FIGURE 3. Survival of $P$. argentinus (cohorts I, II, and III) for males and females respectively (right scale) from initial age to total dilution of the cohorts. Curves 1. II y III: Tails of CR (left scale) in function of the time, according modified of RODRIGUES CAPITULO \& FREYRE, (1989) at Bertalanffy's model.

en la figura 5. Esto se correspondió, con $15 \mathrm{~g}$ (aproximadamente) por unidad de esfuerzo $\left(420 \mathrm{gm}^{-2}\right)$ en la época de menor densidad (invierno) y $110 \mathrm{~g} \mathrm{~m}^{-2}$ en la estación climática de mayor densidad (verano), según puede observarse en la figura 5; esto se corresponde respectivamente con $357 \mathrm{~g} \mathrm{y}$ $2619 \mathrm{~g}$ por Ha. Puede deducirse entonces que el mayor aporte de biomasa corresponde al realizado por las hembras de la segunda cohorte. Ésta, así como la primera y segunda de machos retornan a la zona costera hacia el primer año de vida con una biomasa mayor que la que tenían cuando iniciaron la emigración local en el otoño anterior. Puede interpretarse entonces que los desplazamientos aportan biomasa producida en otras comunidades de la laguna (juncal, potamogeton, etc.) que es consumida en playas libres de vegetación por los depredadores. Las mayores concentraciones de biomasa $P$. argentinus se producen en verano (Fig. 5) y representan en el área de estudio un orden de magnitud mayor que la biomasa de invierno.
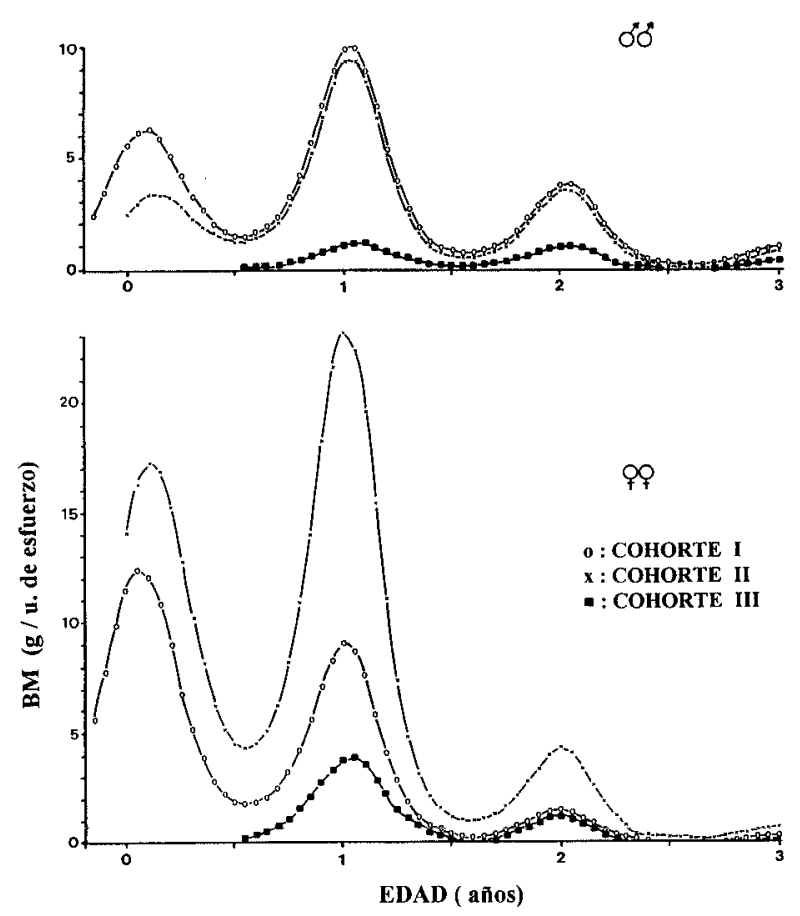

FIGURA 4. (a, b). Biomasa parcial de $P$ argentinus para machos (a) y hembras (b), obtenida a partir del peso formólico medio y $n .{ }^{\circ}$ de individuos estimados para cada cohorte en función del tiempo (referida a la unidad de muestreo).

FIGURE 4. (a, b). Partial biosmass of $P$. argentinis for males (a) and females (b), obtained from mean formaldehyde weight and $n{ }^{\circ}$ of individuals estimated for each cohort in function of the year (referred at the sampling unity).

Fecundidad. La descomposición polimodal correspondiente a las frecuencias de hembras ovígeras a lo largo del año (Fig. 6a) permitió obtener 3 curvas normales cuyos parámetros se muestran en la Tabla II. En la Figura $6 b, c$ y $d$ se muestra el \% acumulado de hembras ovígeras, según los diferentes clases de tamaño, en estos períodos (curvas sigmoides). Cabe mencionar sin embargo que en el tercer desove (Fig. 6 d) la cantidad de hembras maduras participantes apenas llega al $35 \%$, no pudiéndose asimilar el gráfico a una curva sigmoide de porcentajes acumulados. Esta parıcular situación podría explicarse por una mayor extensión del período en la tercera puesta encontrándose hembras ya desovadas, o bien por una menor participación de aquellas en esta etapa. La respuesta a esta incógnita podría darse tras el estudio del ovario en este 


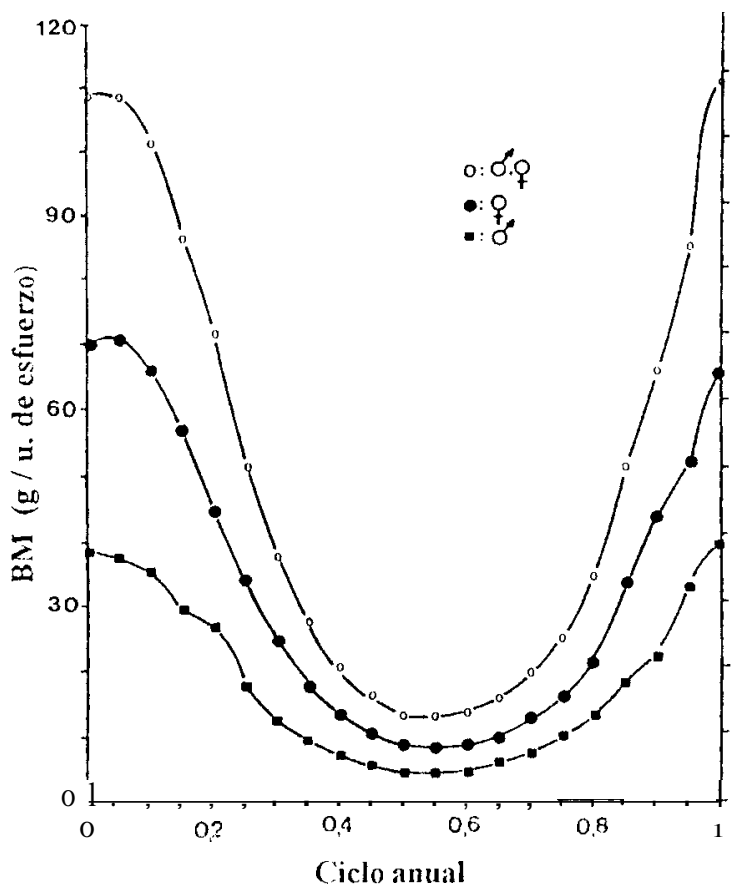

FIGURA 5. Biomasa de machos. hembras y total de P. argentitus en un ciclo anual (referida a la unidad de muestreo).

FIGURE 5. Mnle. female and total biomass of $P$ argentinus in an annual cycle (referred at the sampling unity).

período reproductivo. El análisis posterior del porcentaje de
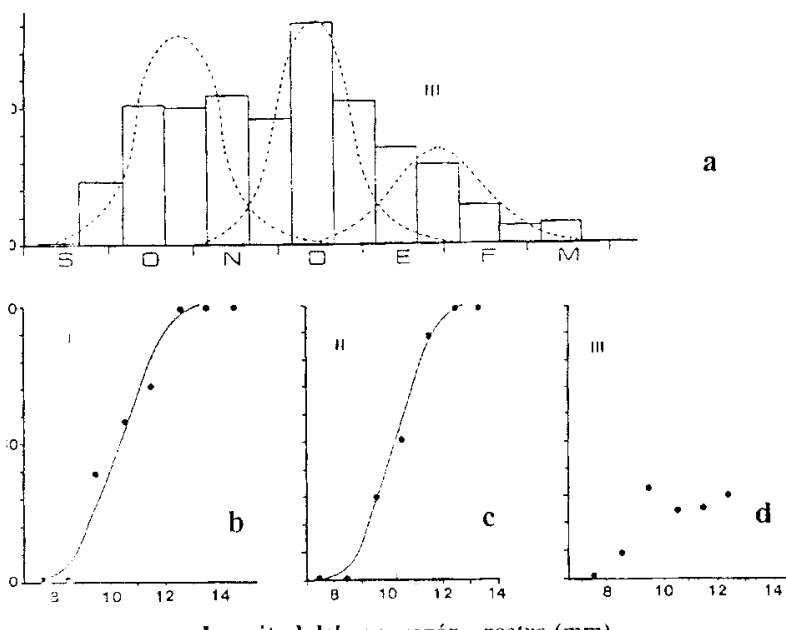

FIGURA 6 a. h. c y d, (a): frecuencia mensual de hembras ovígeras y descomposición polimodal (línea punteada) en relación con las 3 épocas de desove; (b. c. d) $5 \%$ de hembras ovígerns en función de las tallas para los tres desoves. En línea entera se representó la ciirva media asimilada a \% acumulados.

FIGURE 6 a. b. c y d. (a) iiionthly frequencies of the ovigerous frenales and polimodal decomposition (pointed line) in relation with 3 spawning season; (b, c. d) $\%$ of the ovigerous females in relation with the tails for the three spawning In entire line was represented the mean ciirve nssimilated to the accumulated $\%$
TABLA II. Tiempos medios ( $\mathrm{t}$ ) de cada desove (cn unidades de años) y fecha correipondientes, obtenidos a partur de la descomposición polimodal de porcentaje de hembras ovígeras en el período estudiado: (r) coeficiente de coi-relación de la recta prohnbilíitica justa u los datos.

TABLE II. Mean time ( $t$ ) of spawning (year unity) and dates. obtained from the poyniodnl decomposition of the pereent of the ovigerous females in the studied period: (r) coefficient of correlation of the adjusted curve.

\section{Desove $t$ (tiempo) Desv. típica Fecha r}

\begin{tabular}{|lllcc|}
\hline 1 & 0.817 & 0.17 & $24-$ X & -0.99 \\
2 & 0.953 & 0.10 & $13-$ XII & -1.00 \\
3 & 1.078 & 0.11 & $28-I$ & -0.93 \\
\hline
\end{tabular}

hembras ovígeras por clase de tamaño (escala probabilística) para los tres períodos (Fig. 7), dio como resultado una tamaño medio de primera maduración de $10.9 \mathrm{~mm}$ de longitud del caparazón-rostro, correspondiendo a individuos de 1 año de edad aproximadamente.

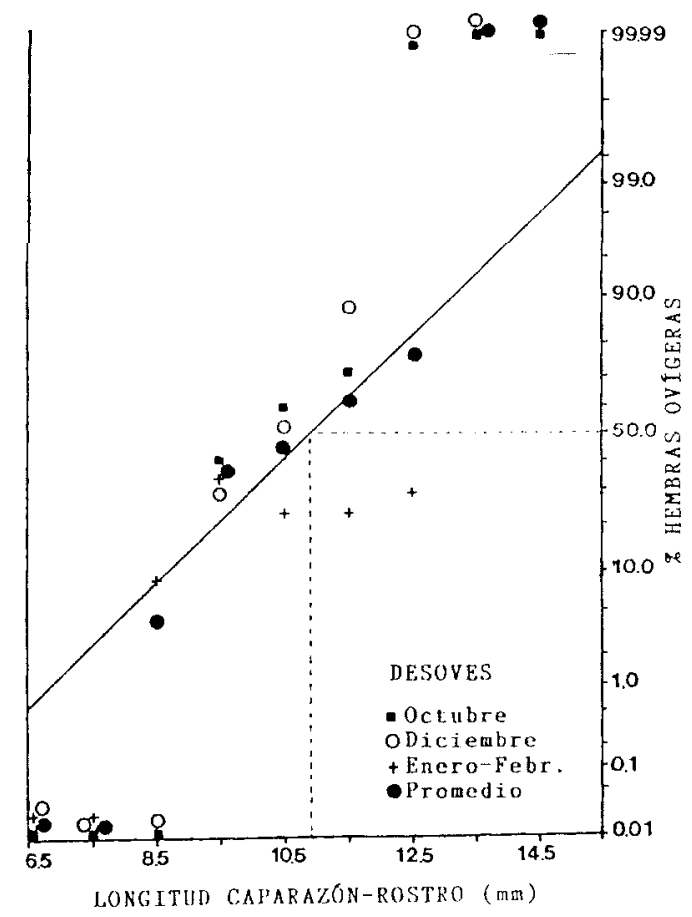

FIGURA 7. Talla media de primera maduración para hembras de P. argentmus en escala probahilística.

FIGURE 7. Mean tail of the first maturation for females of P. argentmus in a probabilistic scale.

El diagrama de dispersión del número de huevos en función de la talla de las hembras ovígeras puede observarse en la Figura 8. La expresión que se ajustó a los valores fue:

$F=0.749 L^{2.014}: r=0.813(n=83)$

Los valores máximos observados correspondieron a 
hembras de $15,5 \mathrm{~mm}$ de caparazón-rostro con 230 embriones sujetos en los pleópodos. El peso fresco medio de los huevos fue $0,876 \mathrm{mg}$ y el volúmen $0,237 \pm 0,026 \mathrm{~mm}$ ' (media \pm desviación típica). Si comparamos la cantidad de huevos sujeta en los pleópodos con la de Macrobrachium borellii, (50-120) la especie de palaemonido con la cual coexiste en ambientes de la Provincia de Buenos Aires (BOSCHI, 1981), observamos que $\mathrm{P}$. argentinus presenta una mayor proporción de embriones.

De acuerdo con los datos de crecimiento según

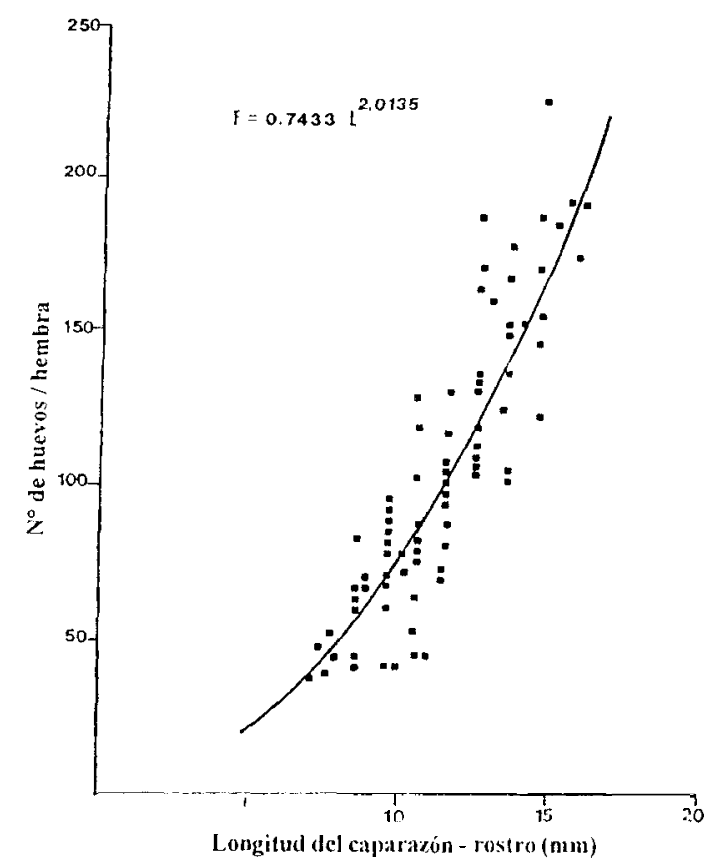

FIGURA 8. Número de embriones sujetos a los pleópodos en relación con la talla del caparazón-rosto de las hembras.

FIGURE 8.. Embryos number subjects to the pleopodes in relation with the tails of the caparison-rostrum of the females.

RODRIGUES CAPITULO \& FREYRE (1989), se obtuvieron las tallas de las hembras considerando las siguientes constantes de crecimiento: $\mathrm{L} \infty=16 \mathrm{~mm} ; \mathrm{k}=1,071 ; \mathrm{t}_{\mathrm{o} 1}=0.507 ; \mathrm{t}_{\mathrm{o} 2}=$ $0.653 ; \mathrm{t}_{\mathrm{o} 3}=1.268$;

donde $\mathrm{L}_{\infty}$ es la longitud infinita, $\mathrm{k}$ la constante de crecimiento $\mathrm{y}_{\mathrm{o}}$ el tiempo inicial de cada cohorte. Luego de establecer los tamaños de las hembras en los 3 momentos de desove máximo se determinó, según el modelo de supervivencia exponencial (modificado según la ecuación (6)), el número de hembras presentes en esos momentos. El gráfico de la figura 6a permitió deducir el porcentaje de esas hembras que participó en la puesta y la recta obtenida a partir del ta ma ño medio de primera maduración en escala probabilística
(Fig. 7),el porcentaje de participación dc acuerdo a la talla de las mismas. El producto de estas dos proporciones multiplicado por la fecundidad, según su expresión exponencial, para las tallas respectivas, permitieron la confección del gráfico de la figura 9, donde se resumen los aportes de cada cohorte en los tres períodos correspondientes a la primavera, verano medio y verano avanzado. Estos resultados estuvieron siempre referidos a la unidad de esfuerzo

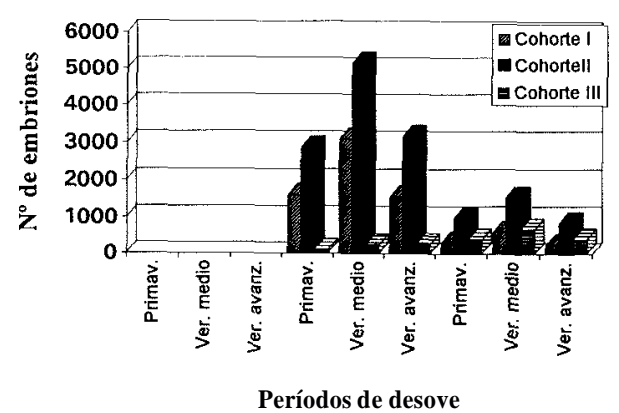

FIGURA 9. Número de embriones aportados por las hembras de P. argentinus de las tres cohortes en las épocas de desove (referidos a la unidad de muestreo considerada).

FIGURE 9. Embryos numbre contiboted for the three female cohorts of $P$. argentinus in the spawning seasons (refrred at the sampling iinity).

equivalente a un lance de pesca. El primer desove se compuso entonces de un total de 6324 embriones, el segundo de 11500 y el tercero de 6650. Comparando estos valores con los nacimientos, estimados por las ecuaciones de supervivencia, se pudo establecer que las hembras están representados en una proporción 2:1 en relación a los machos (Fig. 10).

Según GOLDSTEIN \& LAURIA DE CIDRE (1974) el desove en los camarones del lago de Palermo (Ciudad de Buenos Aires) alcanza su primer máximo en octubre con la casi totalidad de las hembras (93\%) seguido de un segundo desove con menor proporción de hembras ( $38 \%$ ) con un pico en enero. En Chascomús, en cambio, el mayor porcentaje de hembras ovígeras se observó en diciembre (83\%). El desove de primavera sólo alcanzó al $50 \%$ de las hembras. Es posible que esta diferencia a favor de un mayor porcentaje de hembras ovígeras en el primer desove del lago de Palermo se deba a las temperaturas algo más elevadas que se registran allí y que favorecerían la oviposición más temprana. No debe dejar de atenderse sin embargo las posibles variaciones anuales de la población.

BOURDON (1962), señala la existencia de una correlacióri positiva entre la talla de las hembras y el grado de desarrollo de los ovarios. Esto se correlaciona positivamente con la curva 


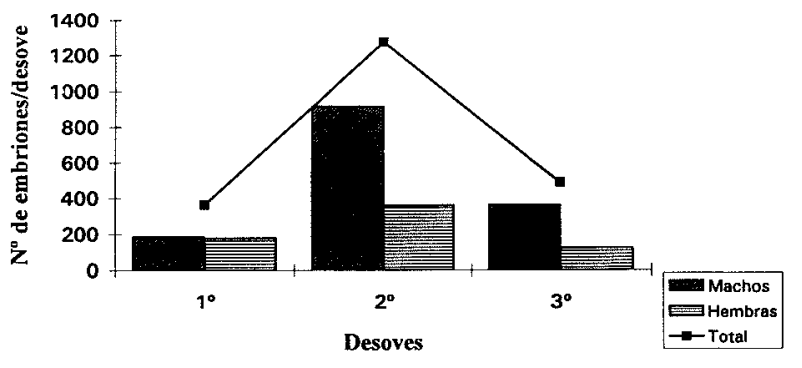

FIGURA 10. Proporción estimada de embriones masculinos y femeninos de P. argentinus en los tres desoves del año.

FIGURE 10. Male and female embryos proportion of P. argentinus in the three spawning of the year.

de fecundidad hallada a través de la ecuación calculada en este trabajo. Según se desprende de nuestras observaciones (Fig. 6) el tamaño medio de primera maduración se alcanza con tallas superiores a $10 \mathrm{~mm}$ de $\mathrm{CR}$; luego la tercera cohorte apenas participa de los desoves de primavera y verano dado que llega con tallas entre 6 y $7 \mathrm{~mm}$ de $\mathrm{CR}$ (hembras inmaduras). Su aporte se hace mas efectivo en otoño, donde la mayoría de las hembras provenientes de las cohortes 1 y 2 ya desovaron. Es muy probable que la baja proporción de hembras ovígeras en esta tercer puesta (Fig. 6 d) se deba a la suma de estos supuestos. Esto también se puede corroborar con lo expuesto por GOLDSTEIN \& LAURIA DE CIDRE (1974) quienes sostuvieron que el $100 \%$ de las hembras púberes completan su gametogénesis en noviembre (Primavera), participando las hembras adultas en los desoves de primera y verano. Es probable que el $13 \%$ de las hembras jóvenes que aún no participan de la reproducción en esos períodos, según estas mismas autoras, se corresponda con ejemplares nacidos en el otoño anterior (cohorte 3) (RODRIGUES CAPITULO \& FREYRE, 1989).

\section{BIBLIOGRAFÍA}

BOSCHI, E., 1961. Sobre el primer estadío larval de dos especies de camarones de agua dulce (Crustacea, Palaemonidae). Actas y Trabajos del Primer Congreso Sudamericano de Zoología, La Plata 2: 69-77.

BOSCHI, E., 1981. Decapoda Natantia. En Fauna de Agua Dulce de la República Argentina. R.A. Ringuelet ed. 26: 61 pp.

BOURDON, 1962. Observations préliminaires sur la ponte des Xanthidae. Bull. Soc. Lorraine Sci. 2: 1-28,

DANGAWS, N.V., 1976. Descrippción sistemática de los parámetros morfométricos considerados en las lagunas pampásicas. Limnobios (La Plata) 1 (2): 35-59.

DESTEFANIS \& FREYRE, 1972. Relaciones tróficas en los peces de la Laguna Chascomús con un intento de referenciación ecológica y tratamiento bioestadístico del espectro trófico. Act. Zool. Lill. 29: 17-33.

FREYRE, L. R., 1975. Normas para el muestreo de un ambiente pesqquero lagunar. M.A.A., Dción. Rec. Pesq., La Plata:

FREYRE, L. R., 1981. Un método semicomputarizado para el análisis de distribuciones polimodales de frecuencias en estudios de dinámica poblacional de peces. Limnobios (La Plata), 2(4): 215-225.

GOLDSTEIN, B. \& LAURIA DE CIDRE, L., 1974. Ciclo de maduración sexual y observaciones preliminares sobre el desove del camarón dulceacuícola Palaemonetes argentinus (Nobili 1901) (Crustacea, Caridea, Palaemonidae) 1. Hembra. Physis (Buenos Aires), B 33 (87): 165-176.

GULLAND, J. A., 1971. Manual de métodos para la evaluación de las poblaciones de las poblaciones de peces. Acrilia FAO, Zaragoza: 164 pp.

MALLO, J. C. \& E. E. BOSCHI, 1982. Contribución al conocimiento del ciclo vital del camarón Peisos petrunkevitchi de la región de Mar del Plata, Argentina (Crustacea, Decapoda, Sergestidae). PHYSIS (Buenos Aires)Secc. A 41 (100): 85-98.

MENU MARQUE, S. A., 1973. Desarrollo larval de Palaemonetes argentinus (Nobili 1901) en el laboratorio (Crustacea, Caridea, Palaemonidae). Physis (Buenos Aires) B 32 (85): 149-169.

RICKER,W.E.,1968. Methods for assesment ofjish production in fresh waters. I.B.P. Handbook, 3. Blackwell sc. publ.: 313 pp.

RICKER, W.E., 1973. Linear regresions in fisheries research. J. Fish. Res. Board. Canada, 30 (3): 409-434.

RINGUELET, R.A., 1949. Camarones y cangrejos de la zona de Goya (Sergestidae Palaemonidae y Trichodactylinae). Notas Mus. La Plata. Univ. Nac. La Plata, 14 (119): 79-109.

RINGUELET, R.A., 1962. Ecología Acuática Continental. Eudeba, Buenos Aires: 138 pp.

RINGUELET, R.A., R. IRIART \& A. ESCALANTE, 1980. Alimentación del pejerrey (Basilicthys bonariensis bonariensis, Atherinidae) en la Laguna Chascomús (Buenos Aires, Argentina). relaciones ccológicas de complementación y eficiencia trófica del plancton. Limnobios (La Plata) 1 (10): 447-460.

RODRIGUES CAPÍTULO, A. \& FREYRE, L.R. , 1979. Metabolismo energético del camarón de agua dulce Palaemonetes argentinus Nobili (Decapoda Natantia Caridea Palaemonidae) de la Laguna Chascomús. Limnobios (La Plata) 1 (9): 337-345.

RODRIGUES CAPÍTULO, A. \& FREYRE, L.R. , 1989. 
Demografía de Palaemonetes (Palaemonetes)argentinus Nobili (Decapoda Natantia. 1. Crecimiento. Limnobios 2 (10): 744-756.

SCHULDT, M., 1980a. La estructura ovárica de Palaemonetes argentinus Nobili, 1901, en relación con aspectos actuales de la morfología funcional en crustáceos superiores. Neotropica (La Plata)26 (76): 155-162.

SCHULDT, M., 1980b. El ovario de Palaemonetes argentinus Nobili, 1901 (Crustacea Palaemonidae). Su comportamiento entre puesta y puesta. Un enfoque estereométrico. Limnobios (La Plata), 2 (1): 23-35.

SCHULDT, M., 1981. Interpretación funcional de cambios microanatómicos en el ovario de Palaemonetes argentinus Nobili, 1901 (Crustacca Palaemonidae) durante desove y post puesta.Limnobios (La Plata), 2 (3): 141-151.

SCHULDT, M. \& A. RODRIGUES CAPÍ TULO, 1985.
Biological and pathological aspects of parasitism in the branchial chamber of Palaemonetes argentinus (Crustacea: Decapoda) by infestation with Probopyrus cf. oviformis (Crustacea: Isopoda). J. Invert. Pathol., 45 (2): 139-146.

SENDRA, E.D. \& L. R. FREYRE, 1978. Dinámica poblacional de Brycorzamericus iheringi (Pisces Tetragonoperidae) de la Laguna Chascomús. Limnobios (La Plata) 1 (8): 299-321.

SENDRA, E.D. \& L. R. FREYRE, 1981. Estudio demográfico de Cheirodon interruptus interruptus (Pisces tetragonopteridae) de la Laguna Chascomús. II. Supervivencia y evaluación de los modelos demográficos. Limnobios (La Plata) 2 (4): 265-272.

WATERMAN, T. H., 1960. The phisiology of Crustacea. Cademic Press, 670 pp. 